\title{
SUR LA COHÉRENCE DU PHÉNOMÈNE DE DIFFUSION MULTIPLE DE LA LUMIĖRE DANS I.A RÉSONANCE OPTIQUE
}

\author{
Par Mle Marie-Anne GUiOChon, MM. Jacques E. BLAMont, Jean BRossel, \\ Laboratoire de Physique de l'École Normale Supérieure, Paris.
}

I. Introduction. - Les expériences que nous décrivons ci-dessous, se rapportent aux propriétés de la résonance optique de la vapeur de mercure excitée optiquement par la raie d'intercombinaison $6^{3} P_{1} \leftarrow 6^{1} S_{0} 2537 \AA$ A. Les résultats présentés se rapportent uniquement aux isotopes pairs. La gamme de température explorée s'étend de $-35^{\circ} \mathrm{C}$ $\grave{a}+20^{\circ} \mathrm{C}$.

A. Polarisation de la raie de résonance. En présence d'un champ magnétique $H_{0}$, cette raie se décompose en 3 composantes Zeeman (cas des isotopes pairs), ainsi que le montre la figure 1.

Les états de polarisation des composantes sont déterminés par les règles de sélection $\Delta m_{J}=0$ vibration $\pi, \Delta m_{J}=+1$ vibration $\sigma^{+}$et $\Delta m_{J}=-1$ vibration $\sigma^{-}$.

L'irradiation optique de la vapeur de mercure en lumière polar sée permet d'exciter sélectivement les sous-niveaux $m_{\boldsymbol{J}}$ de l'état excité [1]; par exemple, si l'on emploie une lumière polarisée dont le vecteur électrique vibre parallèlement au champ $H_{0}$, seule la composante $\pi$ est absorbée et seul le sous-niveau $m_{\boldsymbol{J}}=0$ de l'état $6^{3} P_{1}$ est peuplé. La réémission doit donc uniquement comprendre une composante $\pi$. Sous tous ses aspects possibles, c'est ce phénomène qui est à l'origine de la polarisation de la raie de résonance optique telle qu'elle a été découverte par R. W. Wood [2].

B. Dépolarisation de La raie de résonange. Origine. Diffusion multiple des Photons. - La raie de résonance n'est pas totalement polarisée

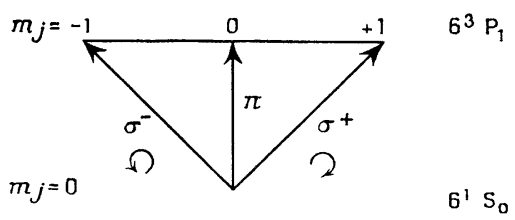

FIG. 1.

dans le mercure naturel. Cela est dû, entre autres, à la présence des isotopes impairs. A cause du spin nucléaire, le diagramme Zeeman de ces isotopes est beaucoup plus complexe que celui de la figure 1 . Cependant, même si l'on tient compte de cet effet, on trouve que le degré de polarisation $P$ de la raie est inférieur à sa valeur théorique $P_{0}$

$$
P<P_{0}
$$

et que, de plus, sa valeur diminue quand la température augmente [3]. Une autre caractéristique très importante de la dépolarisation est qu'elle dépend de façon très critique de la géométrie du montage - conditions d'illumination, volume et forme de la cellule, conditions d'observation. Cette dépolarisation est due à des transferts entre sous-niveaux Zeeman de l'état excité et l'on peut envisager diverses causes pour ce phénomène.

Un grand nombre d'auteurs attribuent la dépolarisation aux "collisions " [4]. Nous pensons que l'importance des collisions a été très largement exagérée et que le phénomène de dépolarisation est presque entièrement dû à la "diffusion multiple » de la lumière de résonance. Celle-ci accompagne la résonance optique chaque fois que la tension de vapeur à laquelle on opère n'est pas très basse.

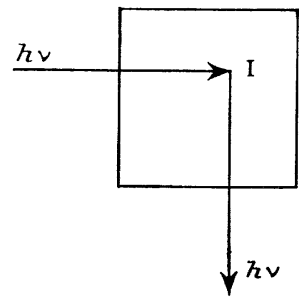

$a$

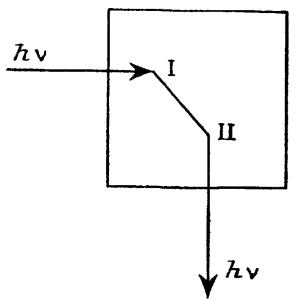

FIg. 2. $b$
La figure $2 a$ illustre la résonance simple et la figure $2 b$ le phénomène de diffusion double.

Si la tension de vapeur est très faible, le photon de résonance absorbé par l'atome $I$ est réémis en moyenne au bout du temps $\tau$ et parvient directement à l'observateur. Au contraire, dans la diffusion double, le photon réémis par l'atome $I$ est absorbé par l'atome $I I$ et n'est détecté qu'après réémission par ce dernier. Un temps de l'ordre de $2 \tau$ sépare maintenant l'absorption initiale de l'émission finale. Plus la diffusion multiple est importante et plus le temps d'emprisonnement $\tau^{\prime}$ est long (on observe facilement des temps $\tau^{\prime}$ de 100 à 1000 fois la durée de vie $\tau$ ). Aux très basses 
densités de vapeur où la diffusion multiple est négligeable, $\tau^{\prime}$ tend vers $\tau[5]$.

La théorie de l'emprisonnement de la raie de résonance a été développée par de nombreux auteurs [6]. Elle montre que si l'on" crée pendant un intervalle de temps très court $(\ll, \tau)$ des atomes dans l'état excité, ces atomes, en retombant à l'état fondamental, émettent la raie de résonance avec une intensité qui décroît en fonction du temps suivant la loi :

$$
I=\sum_{j} I_{j} \mathrm{e}^{-t / \tau_{j}^{\prime}}
$$

ceci diffère en général assez peu d'une expression ne contenant qu'une exponentielle :

$$
I=I_{0} \mathrm{e}^{-t / \tau^{\prime}}
$$

$\tau^{\prime}$ étant de l'ordre du $\tau_{j}^{\prime}$ le plus long ;

$\tau^{\prime}$ tend vers $\tau$ quand la pression est très basse.

L'expérience (Garrett [5], Holstein [6]) confirme très bien les conclusions précédentes. Elle montre que dans le mercure, la diffusion multiple est déjà appréciable à - $15^{\circ} \mathrm{C}$ dans des cellules de quelques centimètres de diamètre. Ia figure 3

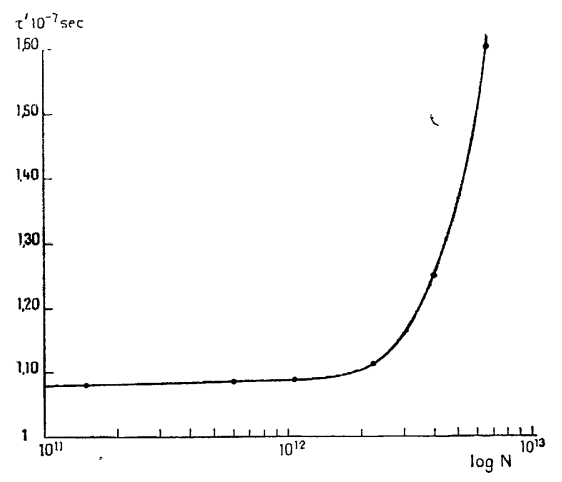

FIG. 3.

représente les résultats de Garrett. En ordonnée $\tau^{\prime}$ en abscisses $\log N(N=$ nombre total d'atomes de mercure par $\mathrm{cm}^{3}$ ). Comme pour le degré de polarisation, la valeur que l'on obtient pour le temps d'emprisonnement dépend beaucoup de la géométrie du montage adopté.

On peut faire l'hypothèse que la dépolarisation est due à des collisions et calculer la section efficace correspondant à ce phénomène. On trouve numériquement des valeurs de l'ordre de $\lambda^{2}$. ( $\lambda$, longueur d'onde de la raie de résonance optique).

On peut, de même, envisager le phénomène d'absorption de la raie de résonance comme une collision entre un atome et son photon de résonance. Si l'on calcule la section efficace correspondante, on trouve encore sensiblement $\lambda^{2}$. Cela indique qu'il n'est pas absurde d'admettre que la dépolarisation soit due à la diffusion multiple. De toute manière, l'énormité des sections efficaces précédentes rend improbable un mécanisme par collisions (au sens de la théorie cinétique). Le fait que la dépolarisation et la diffusion multiple dépendent beaucoup de la géométrie du montage indique clairement que dans les 2 cas " l'interaction » correspondante se produit à de très grandes distances (de l'ordre du millimètre) ce qui semble encore exclure les collisions entre atomes comme cause des 2 processus précédents.

Les expériences de Buhl [7] indiquent clairement aussi que la dépolarisation est due à la diffusion multiple. L'étude de la dépolarisation à des champs magnétiques différents lui a permis de montrer que la loi d'interaction qu'il faut adopter pour rendre compte des résultats expérimentaux est une loi en $1 / r$ et qu'il ne s'agit certainement pas d'une interaction dipôle-dipôle. La loi en $1 / r$ indique que l'interaction se fait par l'intermédiaire du champ de rayonnement. On sait enfin que la dépolarisation croît très vite quand la température augmente : Dans l'hypothèse des collisions cela signifie que le nombre de transferts entre sous-niveaux Zeeman est appréciable dans un temps de l'ordre de la durée de vie $\tau$, donc que le temps moyen entre " collisions " est court par rapport à $\tau$. Au terme de la théorie de Lorentz, il devrait en résulter un élargissement notable du niveau. Nous verrons que l'on observe tout juste le contraire. Il est donc impossible d'attribuer la dépolarisation à des collisions

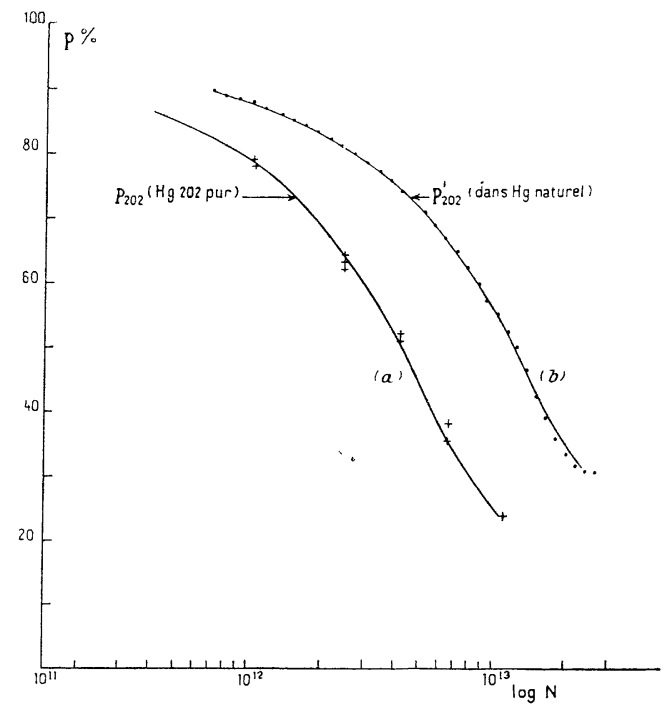

Fig. 4.

On peut montrer directement d'ailleurs qu'entre -30 et $+20^{\circ} \mathrm{C}$ les collisions entre isotopes différents du mercure n'influent pas sur la dépolarisation. Nous avons mesuré dans la gamme de températures précédentes le taux de polarisation $P$ de la vapeur de mercure dans les, conditions ciaprès :

a) Cellule de résonance contenant l'isotope ${ }^{202} \mathrm{Hg}$ pur. Les résultats sont portés sur la figure 4 
(courbe a), en ordonnées $P_{202}$, en abscisses log $N$, $N$ nombre total d'atomes de mercure par $\mathrm{cm}^{3}$.

b) Cellule de résonance contenant du mercure naturel mais excitée par une lampe contenant ${ }^{202} \mathrm{Hg}$ pur (courbe b). - $P_{202}^{\prime}$.

On peut vérifier (fig. 4) que les 2 courbes se déduisent très sensiblement l'une de l'autre par une translation (sur l'axe des abscisses) égale à $\log 1 / 0,29$ (logarithme du rapport des concentrations isotopiques de ${ }^{202} \mathrm{Hg}$ dans la première cellule à celle de ${ }^{202} \mathrm{Hg}$ dans le mercure naturel). En d'autres termes, si l'on porte en abscisses le nombre d'atom es diffusants dans les 2 cas on trouve que les points $P_{202}$ et $P_{202}^{\prime}$ tombent sur la même courbe. A nombre d'atomes diffusants égaux la dépolarisation est la mêre pour ${ }^{202} \mathrm{Hg}$ lorsque les autres isotopes sont absents que pour ${ }^{202} \mathrm{Hg}$ dans le miercure naturel en présence des autres isotopes. Si bien que la dépolarisation de ${ }^{202} \mathrm{Hg}$ dans le mercure naturel est un processus où n'intervient que cet isotope et son propre photon de résonance.

Il n'en est évidemment plus ainsi lorsque les raies de résonance de 2 isotopes coïncident accidentellement. C'est le cas, par exemple, pour les raies dues à ${ }^{198} \mathrm{Hg}$ et à ${ }^{201} \mathrm{Hg}$ (composante $F=3 / 2$ ) dans le mercure naturel. Si l'on excite le mercure naturel par une lampe contenant ${ }^{198} \mathrm{Hg}$ le degré de polarisation de la lumière réémise ne varie pas du tout suivant la courbe de la figure 4.

En résumé nous avons toutes raisons de penser que la dépolarisation comme l'accroissement du temps d'emprisonnement lorsque la tension de vapeur augmente ont pour origine commune la diffusion multiple de la lumière.

II. Mesures de durées de vie. - Il semble enfin qu'il existe toute une classe de phénomènes qui sont gravement affectés par la diffusion multiple : Ce sont ceux qui concernent les mesures de durés de vie $\tau$ par les méthodes conventionnelles de l'optique (absorption, dispersion anormale, dépolarisation accompagnant la rotation du plan de polarisation [8]). On constate une dispersion assez forte des résultats. Lorsque ces mesures ont été conduites à diverses températures, on trouve des variations appréciables. Lorsqu'en fin elles ont pu être effectuées séparément sur les divers isotopes d'un mélange, elles fournissent des "durées de vie " différentes pour les divers isotopes [9]. Nous pensons qu'il s'agit de mesures donnant en fait, un temps d'emprisonnement et qu'il faut extrapoler à des températures assez basses pour obtenir la durée de vie $\tau$.

A. Mesures a partir des méthodes de radioFRÉQUENCE. - Brossel et Bitter [10] ont mesuré la durée de vie au niveau $6^{3} P_{1}$ de $\mathrm{Hg}$ par une méthode complètement différente et qui semblait devoir éviter les inconvénients précédents. Nous résumons ci-dessous le principe de leur méthode ainsi que les résultats auxquels ils étaient parvenus. A l'occasion de recherches sur l'effet Stark du niveau $6^{3} P_{1}$ de $\mathrm{Hg}$ nous avons été conduits à reprendre leurs expériences. Nous sommes arrivés à la conclusion que cette méthode aboutit, elle aussi, à la mesure d'un temps d'emprisonnement $T_{\mathrm{e}}$. Nos mesures nous permettent en outre de mettre nettement en évid znce une propriété très importante de la diffusion multiple, celle d'être un Processus CoHÉrENT.

Brossel et Bitter provoquent l'excitation optique de la vapeur de mercure avec la raie $2537 \AA$ polarisée en $\pi$. Seul le niveau médian supérieur $m_{J}=0$ est excité ( $f i g .1$ ) et, en l'absence des causes de dépolarisation analysées précédemment, la lumière réémise est polarisée linéairement en $\pi$. Si un champ de radiofréquence (perpendiculaire à $H_{0}$ ) induit des transitions vers les niveaux $m_{\boldsymbol{J}}= \pm 1$, des composantes $\sigma$ apparaissent et l'on observe une dépolarisation de la lumière réémise.

On peut calculer l'intensité $B$ de la lumière $\sigma$ ainsi produite en fonction de :

$H_{1}$ : amplitude du champ haute fréquence de fréquence $\nu$ induisant les transitions $(\omega=2 \pi \nu)$;

$\gamma$ : rapport gyro-magnétique du niveau $6^{3} P_{1}$;

$T_{\mathrm{e}}$ : «durée de vie » de ce niveau ;

$\omega_{0}=2 \pi \nu_{0}: \nu_{0}$ fréquence de la transition

$m_{J}=0 \rightarrow m_{J}= \pm 1$

$\Omega=\omega-\omega_{0}$.

On trouve

$B\left(\gamma H_{1}, T_{\mathrm{e}}, \Omega\right) \equiv \frac{\left(\gamma H_{1}\right)^{2}}{\left(\gamma H_{1}\right)^{2}+\Omega^{2}}$

$\left[\frac{\left(\gamma H_{1}\right)^{2}}{\left(\frac{1}{T_{\mathrm{e}}}\right)^{2}+4\left(\gamma H_{1}^{2}\right)+4 \Omega^{2}}+\frac{\Omega_{1}^{2}}{\left(\frac{1}{T_{\mathrm{e}}}\right)^{2}+\left(\gamma H_{1}\right)^{2}+\Omega^{2}}\right]$.

Le calcul précédent est basé sur l'hypothèse qu'aucune perturbation autre que $H_{1}$ n'agit sur l'atome quand il est dans l'état excité. Ainsi, la forme de la raie n'est $p$ as donnée par une formule de type Lorentz. Cela provient de ce que le champ de radiofréquence met en intercommunication 3 niveaux énergétiques équidistants. Le champ $H_{1}$ produit la réorientation du moment magnétique, phénomène pour lequel les probabilités de transition ont étécalculées par Majorana [11]. La courbe précédente est une courbe en cloche lorsque $\gamma H_{1}<\frac{1}{\sqrt{8}}$. Au contraire, pour les fortes valeurs de $H_{1}$ on obtient une courbe renversée au centre. La propriété résulte de la formule de Majorana et est due à l'existence de transitions directes à 2 quanta entre les niveaux \pm 1 . De telles courbes sont représentées figure 6 .

A résonance $\Omega=\omega-\omega_{0}=0$, la quantité $y$

$$
y=\frac{\gamma^{2} H_{1}^{2}}{B\left(\gamma H_{1}, T_{\mathrm{e}}, 0\right)}=4 \gamma^{2} H_{1}^{2}+\frac{1}{T_{\mathrm{e}}{ }^{2}}
$$

est une fonction linéaire de $H_{1}^{2}$. 
La largeur $\Delta \omega$ de la raie, déduite de la formule (1) est donnée par l'expression approchée :

$$
\Delta \omega^{2}=\frac{4}{T_{\mathrm{e}}{ }^{2}}\left(1+5,8 \gamma^{2} H_{1}^{2} T_{\mathrm{e}}{ }^{2}\right) .
$$

Aux faibles valeurs de $\gamma H_{1}, \Delta \omega=2 / T_{\mathrm{e}}$ : la largeur de la raie est la somme des largeurs des niveaux de départ et d'arrivée de la transition. Le terme d'élargissement $5,8\left(\gamma H_{1} T_{\mathrm{e}}\right)^{2}$ vient de ce que l'émission induite par le champ $H_{1}$ n'est plus négligeable devant l'émission spontanée optique. Les formules 1,2 et 3 sont très bien vérifiées par l'expérience. Elles permettent de mesurer le paramètre $T_{\mathrm{e}}$ que Brossel et Bitter interprétent comme une durée de vie. Ils trouvent, en opérant à $0{ }^{\circ} \mathrm{C}$ sur le mercure naturel et en faisant la mesure sur l'ensemble des isotopes pairs que $T_{\mathrm{e}}=1,5510^{-7} \mathrm{sec}=T_{\mathrm{e} 0}$. Leur source avait une émission de type "broad line" sur l'ensemble de la structure hyperfine.

A la même température une mesure analogue fut effectuée sur ${ }^{199} \mathrm{Hg}$. L'échantillon utilisé contenait $60 \%$ de cet isotope. La résonance $F=3 / 2$ fournissait $T_{\mathrm{e}}=1,4510^{-7} \mathrm{sec}$.

De même les résonances $F=3 / 2$ de ${ }^{201} \mathrm{Hg}$ donnent $T_{\mathrm{e}}=1,55.10^{-7} \mathrm{sec}$ avec un échantillon contenant $60 \%$ de cet isotope. (Soulignons que dans ce dernier cas, les formes de raie sont des formes de Lorentz : du fait du découplage I, J, le champ de radiofréquence met en intercommunication 2 niveaux énergétiques seulement). Il semblait très satisfaisant de trouver la même valeur pour tous les isotopes (il s'agit, en fait, d'une coïncidence accidentelle). Mais aucune interprétation n'était proposée pour expliquer la différence entre cette valeur, et celle résultant de déterminations de type classique $\left(1,14.10^{-7}\right.$ sec $)$. Il semblait donc que la méthode actuelle devait être préférée, parce qu'elle évitait beaucoup des difficultés rencontrées précédemment et que l'interprétation théorique était remarquablement confirmée par l'expérience.

B. Résultats nouveaux. - (Variation de $T_{\mathrm{e}}$ avec la géométrie du montage, la nature de la source excitatrice, la température.)

Nous avons repris la vérification de la formule 1 sur une cellule présentant des caractéristiques géométriques différentes de celles de Brossel-Bitter. En outre, la source utilisée n'était évidemment pas la même. Nous avons pu confirmer complètement leurs résultats : la forme des raies est très bien représentée, dans tous les cas étudiés par la formule 1. Mais pour rendre compte de nos mesures, nous sommes conduits $\grave{a}$ adopter une valeur $d u$ parmètre $T_{\mathrm{e}}$ qui est très différente de la valeur précédente Elle dépend de la source excitatrice et des caractéristiques géométriques de la cellule.

INFLUENCE DE LA GÉOMÉTRIE DU MONTAGE. Par exemple avec une cellule donnée, à $0^{\circ} \mathrm{C}$, en faisant une mesure sur l'ensemble des isotopes pairs on trouve $T_{\mathrm{e}}=1,6510^{-7}$ sec pour une excitation de type broad line et $T_{\mathrm{e}}=1,8010^{-7}$ sec pour une excitation du type narrow line. Avec une source donnée, (narrow line) une cellule de dimensions $20 \mathrm{~mm} \times 20 \mathrm{~mm} \times 4 \mathrm{~mm}$ donne $T_{\mathrm{e}}=1,4210^{-7}$ et une de dimensions $35 \mathrm{~mm} \times 40 \mathrm{~mm} \times 7 \mathrm{~mm}$ donne $T_{\mathrm{e}}=1,7210^{-7}$. La raie est plus large pour la cellule plus petite c'est-à-dire pour laquelle la diffusion multiple est la moins importante.

Ainsi, il apparaît à l'évidence que le paramètre $T_{\mathrm{e}}$ ne mesure pas la durée de vie.

INFLUENCE DE LA TEMPÉrature. - Une étude de $T_{\mathrm{e}}$ en fonction de la température confirme ce résultat. La figure 5 , courbe II représente le résultat de nos mesures effectuées entre - 35 et $+20^{\circ} \mathrm{C}$. En ordonnées $T_{\mathrm{e}}$, en abscisses $\log N$ du nombre total d'atomes de mercure par $\mathrm{cm}^{3}$ à la température où l'on opère. La courbe II est obtenue en mesurant $T_{\mathrm{e}}$ sur l'ensemble des isotopes pairs, soit $T_{\mathrm{e}_{0}}$, avec une excitation de type narrow line.

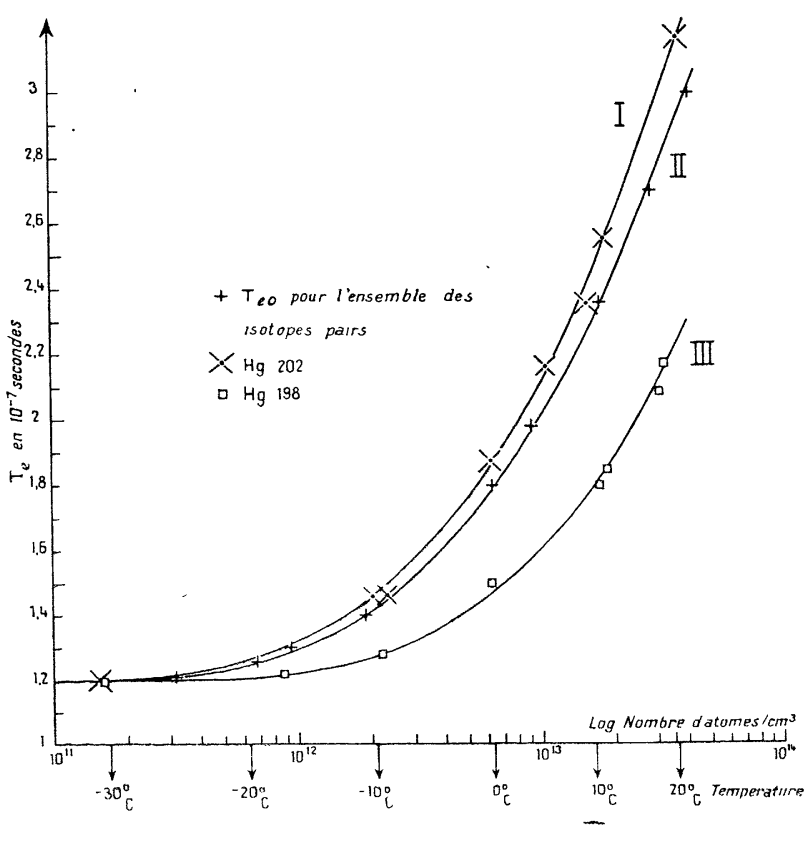

Fig. 5.

On reinarque les points suivants :

a) Qualitativement les variations de $T_{\mathrm{e}_{0}}$ sont tout à fait analogues à celles de $\tau^{\prime}$, le temps d'emprisonnement mesuré par Garrett [5] (fig. 3). Les valeurs obtenues sont très voisines, ce qui est assez remarquable étant donné la difficulté des mesures de Garrett.

b) $T_{\mathrm{e}_{0}}$ croît quand la température augmente. $\left(2,5\right.$ fois plus long à $+20^{\circ}$ qu'à $\left.-35^{\circ} \mathrm{C}\right)$ c'est-àdire que l'on obtient des raies de résonance magnétique, qui, à $+20^{\circ} \mathrm{C}$ sont plusieurs fois plus fines 
que ce que la largeur naturelle des niveaux permet de prévoir. L'effet ressort clairement de la figure 6 . Elle représente des réseaux de résonance magnétique obtenus sur l'ensemble des isotopes pairs dans une cuve contenant du mercure naturel, aux températures $-31^{\circ} \mathrm{C}, 0^{\circ} \mathrm{C}$ et $+22^{\circ} \mathrm{C}$. L'échelle des abscisses est la même pour chacune de 3 courbes. Les courbes sont tracées pour des puissances croissantes de radiofréquence, Nous avons expliqué ci-dessus, l'origine $d u$ renversement observé pour les fortes valeurs de $\gamma H_{1}$. Les courbes à $-31{ }^{\circ} \mathrm{C}$ et $0^{\circ}$ ont été tracées aux mêmes intensités de R. F. et se correspondent 2 à 2 , tandis que la courbe la plus haute du réseau $+22{ }^{\circ} \mathrm{C}$ a été tracée avec la même intensité $\gamma H_{1}$ que la $4 \mathrm{e}$ courbe (à partir du haut) des réseaux à $-31{ }^{\circ} \mathrm{C}$ et $0{ }^{\circ} \mathrm{C}$ (l'amplitude $\gamma H_{1}$ est indiquée par le nombre porté au voisinage de chaque courbe).

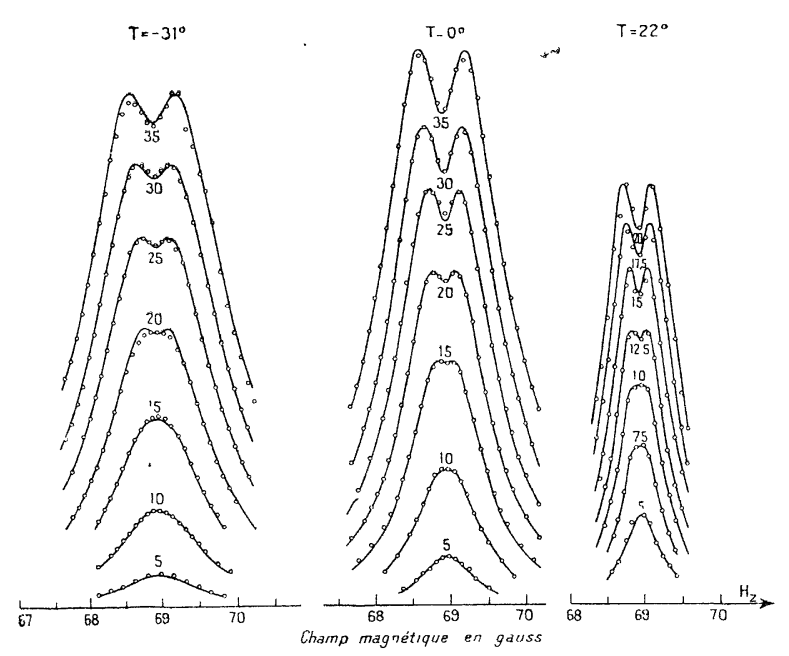

Fig. 6.

Les courbes tracées sont en fait les courbes théoriques résultant de la formule 1 . Les points sont les points expérimentaux. On voit qu'd toute température la vérification de la formule 1 est excellente.

c) Au-dessous de $-20^{\circ} \mathrm{C}$ dans les cellules utilisées, $T_{\mathrm{e}_{0}}$ demeure constant. A ces températures, collisions ou diffusion multiplie jouent un rôle très faible. Nous pensons que la limite ainsi atteinte $\tau=1,2010^{-7} \mathrm{sec}$ représente la durée de vie $d u$ niveau $6^{3} P_{1}$. Cette valeur est très voisine de celle obtenue par Ladenburg [12] $\left(1,14.10^{-7} \mathrm{sec}\right)$ par des mesures de dispersion anormale sur les ailes de la raie d'absorption et de celle obtenue par Garrett [5] $\left(1,08 \cdot 10^{-7} \mathrm{sec}\right)$.

Une autre confirmation du fait que $T_{\mathrm{e}_{0}}$ tend vers $\tau$ est apportée par les mesures suivantes. La cuve contenant du mercure naturel, nous l'excitons avec une lampe de ${ }^{202} \mathrm{Hg}$ pur. Seul l'isotope ${ }^{202} \mathrm{Hg}$ est alors excité et la résonance magnétique observée est celle due à l'isotope ${ }^{202} \mathrm{Hg}$ seul, en présence des autres isotopes. On peut ainsi mesurer $T_{\mathbf{e}_{202}}$ (courbe I, fig. 5). Nous avons fait de même la mesure de $T_{\mathrm{e}_{198}}$ en substituant à la précédente une source contenant ${ }^{198} \mathrm{Hg}$ pur (courbe III).

On voit immédiatement qu'à une température donnée : $T_{\mathrm{e}_{\mathbf{2 0 2}}}>T_{\mathrm{e}_{198}}$. Les raies de résonance magnétique de l'isotope le plus concentré sont les plus fines. (C'est aussi pour cet isotope que la diffusion multiple est la plus importante.)

$\mathrm{Au}$-dessous de $-20^{\circ} \mathrm{C}$

$$
T_{\mathrm{e}_{\mathbf{2 0 2}}}=T_{\mathrm{e}_{\mathbf{1 9 8}}}=T_{\mathrm{e}_{\mathbf{0}}}=\tau \text {. }
$$

Donc $\tau$ est le même pour tous les isotopes pairs.

A une température déterminée le réseau relatif à un isotope pair pur est représenté ici encore par les formules 1 de Majorana-Brossel. Il est évident, dans ces conditions, que la courbe de résonance observée pour l'ensemble des isotopes pairs est la superposition de courbes de résonance indépendantes relatives à chaque isotope. A première vue, il est donc extrêmement suprenant que le réseau relatif à l'ensemble des isotopes pairs vérifie de façon très satisfaisante le réseau théorique résultant de la formule 1 de Majorana-Brossel. Il est naturel, dans ces conditions, que $T_{\mathbf{e}_{0}}$ soit compris entre $T_{\mathrm{e}_{198}}$ et $T_{\mathrm{e}_{202}}$. Quand, en outre, l'on passe d'une excitation "broad line " à une excitation "narrow line », on accroît l'importance des isotopes les plus concentrés $\left({ }^{202} \mathrm{Hg}\right.$ et $\left.{ }^{200} \mathrm{Hg}\right)$ par rapport aux autres $\left({ }^{198} \mathrm{Hg},{ }^{204} \mathrm{Hg}\right)$ c'est-à-dire l'influence des isotopes qui donnent des résonances plus fines. C'est la raison pour laquelle $T_{\mathbf{e}_{0}}$ mesuré avec une excitation " narrow line " est beaucoup plus long que $T_{\mathrm{e}_{0}}$ mesuré avec une excitation " broad line ".

Mais, la propriété la plus intéressante des courbes I et III, figure 5, est la suivante (dans les limites des températures où notre étude a été effectuée) : elles se déduisent l'une de l'autre par une translation sur l'axe des abscisses égale au logarithme du rapport des concentrations isotopiques de ${ }^{202} \mathrm{Hg}$ et ${ }^{198} \mathrm{Hg}(\log 29 / 10)$. Le raisonnement que nous avons fait au sujet du degré de polarisation peut se répéter ici : les points représentatifs tombent sur la même courbe si l'on porte en abscisses le nombre d'atomes diffusants : il s'ensuit que les mécanismes qui déterminent la grandeur de $T_{\mathrm{e}_{202}}$ ou $T_{\mathrm{e}_{198}}$ sont indépendants de la concentration des autres isotopes (entre $-35^{\circ}$ et $+20^{\circ} \mathrm{C}$ ). Les processus qui déterminent $T_{\mathrm{e}}$ pour un isotope déterminé ne font intervenir que cet isotope et son propre photon de résonance. On peut envisager 2 tels processus :

a) Les collisions, au sens de Lorentz [13], entre atomes identiques. Ce processus peut être à l'origine d'une dépolarisation, mais il ne peut qu'aboutir au raccourcissement du paramètre $T_{\mathrm{e}}$ (élargissement de la raie).

$b$ ) La diffusion multiple. Nous verrons ci-dessous comment une diffusion multiple de type cohérent. 
peut expliquer un affinement des raies quand la tension de vapeur augmente. Ce dernier phénomène est donc celui qui l'emporte sur l'autre. Si elles jouent un rôle, les collisions entre atomes identiques jouent un rôle bien moins important que la diffusion multiple cohérente dans la détermination de $T_{\mathrm{e}}$ (entre -35 et $+20^{\circ} \mathrm{C}$ ).

Calcul du paramètre $T_{\mathrm{e}_{0}}$ relatif a l'enSEMBLE DES ISOTOPES PAIRS A PARTIR DE $T_{\mathrm{e}_{202}}$. Nous revenons maintenant au problème de la détermination de $T_{\mathbf{e}_{0}}$ (pour l'ensemble des isotopes pairs) à partir de la courbe I, figure 5 donnant $T_{\mathbf{e}_{\mathbf{2 0 2}}}$ (pour un isotope pair donné) en fonction du nombre d'atomes de cet isotope. Le réseau $A_{0}\left(\gamma H_{1}, T_{\mathbf{e}_{0}}, \Omega\right)$ relatif à l'ensemble des isotopes pairs est la superposition, avec des intensités convenables des réseaux des divers isotopes 204, 202, 200, 198. Cette hypothèse est justifiée par les mesures qui ont montré l'indépendance complète de comportement des divers isotopes.

Nous supposons ensuite que les courbes de résonance d'un isotope donné sont fournies par la formule 1 de Majorana-Brossel. Les mesures relatives au réseau d'un isotope pair dans le mercure naturel justifient complètement cette hypothèse. Les valeurs de $T_{\text {ej }}$ à adopter dans l'expression de $B_{\mathrm{j}}\left(\gamma H_{1}, T_{\mathrm{e} j}, \Omega\right)$ est celle fournie par la courbe I, figure $5, N$ étant déterminé à partir de la concentration de l'isotope envisagé à la température où l'on opère.

Nous écrivons alors

$$
A_{\mathrm{o}}\left(\gamma H_{1}, T_{\mathrm{e}_{\mathbf{0}}}, \Omega\right) \equiv \sum_{\mathbf{j}} a_{\mathrm{j}} b_{\mathrm{j}} B_{\mathrm{j}}\left(\gamma H_{1}, T_{\mathrm{e}_{\mathbf{j}}}, \Omega\right) .
$$

Nous avons souligné plus haut le fait expérimental que nous cherchons à justifier ici, que $A_{0}$ est en fait un réseau de Majorana-Brossel représenté par une équation de type I dans lequel on doit donner à $T_{\mathrm{e}}$ la valeur $T_{\mathrm{e}_{0}}$.

$a_{\mathrm{j}}$ est un facteur caractéristique de la source; il mesure l'intensité avec laquelle la raie de résonance de l'isotope $\mathrm{j}$ est émise par la lampe excitatrice. $a_{\mathrm{j}}$ vaut 1 quel que soit $\mathrm{j}$ pour une excitation " broad line " et est égal à l'intensité relative des composantes hyperfines de la raie 2537 pour une excitation " narrow line ».

$b_{\mathrm{j}}$ est un facteur ne contenant ni $\gamma H_{1}$, ni $\Omega$, et qui fait intervenir divers phénomènes intervenant dans la cuve de résonance; il dépend essentiellement de la température.

Avant d'atteindre la région d'observation la lumière excitatrice traverse une couche de vapeur I et l'observation se fait à travers une couche de vapeur II (fig. 7). Quand on fait varier la température le faisceau excitateur au niveau de la région d'observation est donc plus ou moins affaibli. A une température donnée l'intensité excitatrice de l'isotope le plus concentré est plus affaiblie que celle de l'isotope le plus pauvre. Un deuxième facteur tient à la variation du degré de polarisation $P$ en fonction de la température ou, encore, à une température donnée, en fonction de la concentration iso-

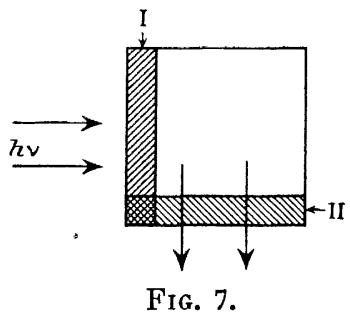

topique. La raie de résonance de l'isotope le plus concentré est plus dépolarisée que celle d'un isotope pauvre ( $f i g .4$ ). Ceci signifie que les différénces de population entre le niveau $m_{\boldsymbol{J}}=0$ et $m_{\boldsymbol{J}}= \pm 1$ sont plus prononcées à cette température, pour l'isotope rare que pour l'isotope abondant. L'intensité de la résonance magnétique est proportionnelle à cette différence de population. Nous incluons dans le facteur $b_{\mathrm{j}}$ tous les phénomènes précédents. Nous donnons dans un appendice la méthode utilisée pour obtenir les valeurs des coefficients $b_{\mathrm{j}}$ ainsi que celle de tous les autres paramètres $\left(T_{\mathrm{ej}}, \gamma H_{1}\right)$.

Tous les paramètres étant connus, nous avons alors procédé à la somme [4] et tracé les réseaux correspondants dans le cas d'une excitation "broad line " et " narrow line " pour la "température $0^{\circ}$. Nous désignons par $\mathrm{S}$ le réseau "somme " ainsi obtenu. Sur le réseau $\mathrm{S}$ ainsi dessiné nous avons cherché à voir si des formules de type 2 et 3 étaient valables. Elles se vérifient très bien. On :peut en particulier mesurer sur le réseau ainsi tracé la valeur $T_{\mathrm{e}_{0}}$ (dans les 2 cas précédents); cette valeur est entièrement déduite de la valeur $T_{\mathrm{e}_{202}}$ à la même température; nous la désignons par $T_{\mathbf{e}_{\mathbf{0}}}$ calculé. Nous les donnons, dans le tableau 1 avec, en regard, les valeurs obtenues par mesure directe sur l'ensemble des isotopes pairs du mercure. L'accord est excellent.

\section{TABLEAU 1}

Broad line ....

Narrow line...

$$
\begin{gathered}
T \mathrm{e}_{0} \text { MESURÉ } \\
\begin{array}{c}
1,6510^{-7} \\
1,8010^{-7}
\end{array}
\end{gathered}
$$

$$
\begin{gathered}
T \mathrm{e}_{0} \text { Caldulfí } \\
\text { 1,6810-7 } \\
1,7510^{-7}
\end{gathered}
$$

Nous avons alors calculé dans les 2 cas le réseau de Majorana-Brossel $U$ en prenant pour le paramètre $T_{\mathrm{e}}$ les valeurs $T_{\mathrm{e}_{0}}$ calc précédentes et à $\gamma H_{1}$, les valeurs déduites de la formule 2 sur le réseau $S$. Il est pratiquement impossible de trouver de différence mesurable entre les réseaux $S$ et $U$. Sur la figure 8, les courbes représentent le réseau $U$, les points représentent le réseau $\mathrm{S}$ (cas de l'excitation «narrow line »). Il est donc impossible de distinguer expérimentalement entre un réseau de Majorana-Brossel 
unique et une somme de tels réseaux de largeurs différentes quand les paramètres changent dans les limites précédemment précisées. Mais cela nous indique clairement que le réseau relatif à un isotope pair pur, peut fort bien être, en fait, une somme de réseaux de largeurs différentes. On sait que lorsque la diffusion multiple est importante, l'émission de lumière de la cellule se fait suivant la loi :

$$
I=\sum_{\mathbf{i}} I_{\mathbf{i}} \mathrm{e}^{-t / T_{\mathbf{e i}}}
$$

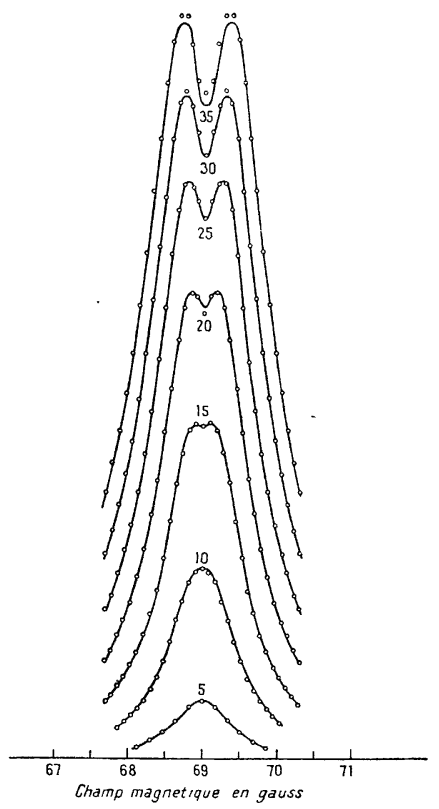

Fig. 8.

Le calcul précédent montre qu'il est tout à fait possible que le réseau observé, corresponde, en fait, à une émission faisant intervenir plusieurs constantes $T_{\mathrm{e}_{\mathrm{i}}}$ (diffusion simple, double, etc.) ce qui est le cas pour la diffusion multiple cohérente.

III. Diffusion multiple cohérente et incohérente. Interprétation des résultats. - Il nous reste maintenant à montrer comment le phénomène de diffusion multiple permet d'expliquer un affinement des raies de résonance magnétique et d obtenir des largeurs inférieures à la largeur naturelle.

Il faut ici distinguer 2 types de diffusion multiple : la diffusion cohérente et là diffusion incohérente.

Envisageons le cas de la diffusion double : le photon incident venant de la source est absorbé por l'atome $I$, réémis en moyenne après un temps $\tau$ égal à la durée de vie, absorbé par l'atome $I I$, réémis de nouveau et détecté par l'observateur (temps d'emprisonnement $\tau^{\prime}$ de l'ordre de $2 \tau$ ). En présence d'un champ de radiofréquence (perturbation dépendant du temps) la fonction d'onde de l'atome $I$ (isotope pair du mercure) est, à l'instant $\theta$ après l'absorption du photon initial

$$
\Psi^{I}(\theta)=C_{+1}(\theta) \Psi_{1}^{I}+C_{0}(\theta) \Psi_{0}^{I}+C_{-1}(\theta) \Psi_{-1}^{I}
$$

$\Psi_{1}^{I}, \Psi_{0}^{I}, \Psi_{-1}^{I}$, étant les états propres de l'atome $I$.

Pour décrire l'évolution de l'atome $I I$, on peut écrire une équation analogue. Quand il faut écrire les conditions initiales, c'est-à-dire la forme que prend la fonction d'onde $\Psi^{\boldsymbol{I I}}$ à l'instant qui suit immédiatement l'absorption du photon par l'atome $I I$ on peut envisager 2 cas :

a) $\Psi^{I I}(\theta)$ est un état propre pur de l'atome II. Dans ce cas, nous dirons que la diffusion est incohérente. On peut considérer que les processus $I$ et $I I$ sont distincts et indépendants. C'est le cas par exemple lorsque les atomes $I$ et $I I$ étant des isotopes différents, une coïncidence accidentelle fait qu'une fréquence propre de $I$ coïncide avec une fréquence propre de $I I$ grâce par exemple à l'effet Doppler dans le mouvement relatif des 2 atomes.

Dans le cas où les atomes $I$ et $I I$ sont identiques et qu'ils ne se trouvent pas en mouvement relatif, toutes leurs fréquences propres coïncident. L'hypothèse de la diffusion incohérente consiste à considérer que l'atome $I I$ est excité par 3 sortes de lumières $\sigma^{+}, \pi$ et $\sigma^{-}$émises par la source $\mathrm{I}$ avec les intensités respectives $\left|C_{1}(\theta)\right|^{2},\left|C_{0}(\theta)\right|^{2},\left|C_{-1}(\theta)\right|^{2}$ et à considérer les 2 processus comme complètement indépendants.

Une diffusion incohérente de ce type est dépolarisante et accroît le temps d'emprisonnement, mais elle conduit nécessairement à un élargissement de la raie de résonance magnétique.

$\mathrm{Au}$ cours d'une résonance magnétique sur l'atome $I$, si les atomes $I$ et $I I$ sont différents, l'atome $I I$ n'intervient pas : il réfléchit fidèlement les changements de polarisation qu'il reçoit, la raie observée a la largeur naturelle.

Si les atomes $I$ et $I I$ sont identiques, l'observateur voit la résonance de l'atome $I I$ éclairé par l'atome $I$, c'est-à-dire par de la lumière qui est d'autant plus dépolarisée que l'on se trouve plus près de la résonance. (La situation est analogue à celle que l'on obtiendrait dans l'expérience suivante : on observe la résonance magnétique d'un atome $I I$, mais au lieu d'exciter cet atome toujours en lumière $\pi$, on éclairerait cet atome avec une proportion croissante de lumière $\sigma$ au fur et à mesure que l'on s'approche de la résonance, ceci à cause de la résonance propre de l'atome I.) Ainsi, dans l'hypothèse de la diffusion incohérente par atomes identiques, le signal de résonance magnétique émis par $I I$ est d'autant plus petit par rapport au signal que $I I$ devrait émettre, que l'on se trouve plus près de la condition de résonance. La résonance est donc plus large qu'elle ne devrait être.

Ainsi, en aucun cas dans cette hypothèse, ne peut-on observer de largeur de raie qui soit inférieure à la largeur naturelle. La raie est d'autant plus 
large que la diffusion multiple est plus importante. Cela est contraire aux observations. Or il est établi que la diffusion multiple existe dans les conditions où nous opérons.

b) $\Psi^{I I}(\theta)$ est de la forme

$$
\Psi^{I I}(\theta)=\underset{\mathbf{i}}{\Sigma} C_{\mathbf{i}}^{\prime}(\theta) \Psi_{\mathbf{i}}^{I I}
$$

dans le cas de 2 isotopes pairs identiques, par exemple

$$
\Psi^{I I}(\theta) \equiv C_{1}^{\prime}(\theta) \Psi_{1}^{I}+C_{0}^{\prime}(\theta) \Psi_{0}^{I}+C_{-1}^{\prime}(\theta) \Psi_{-1}^{I}
$$

expression dans laquelle $C_{1}^{\prime}(\theta), C_{0}^{\prime}(\theta), C_{-1}^{\prime}(\theta)$, ont des grandeurs et des phases relatives déterminées par $C_{1}(\theta), C_{0}(\theta)$ et $C_{-1}(\theta)$ (en tenant compte par exemple de la position relative des atomes $I$ et $I I$ dans le champ $H_{0}$ ).

Nous disons alors que la diffusion est cohérente. Il faut envisager en quelque sorte que l'atome $I I$ est éclairé par un mélange cohérent de photons venant de $I$. (Un exemple connu [14] de ce cas est celui ou $C_{1}=C_{-1}, C_{0}=0$ et que l'on réalise expérimentalement en excitant un atome dans un champ magnétique $H_{0}$ très faible par de la lumière linéaire vibrant perpendiculairement à $H_{0}$ (excitation cohérente en $\sigma^{+}$et $\left.\sigma^{-}\right)$).

On voit facilement, sur un cas particulier, que la diffusion cohérente conduit à un affinement de la raie de résonance magnétique. C'est le cas où $C_{1}^{\prime}=C_{1}, C_{0}^{\prime}=C_{0}, C_{-1}^{\prime}=C_{-1}$, où les atomes $I$ et $I I$ échangent en quelque sorte leur fonction d'onde.

A partir de l'instant $\theta$, l'évolution de la fonction d'onde $\Psi^{I I}$ aux instant $\theta+\theta^{\prime}$ ultérieurs, sera identique à ce qu'aurait été l'évolution de la fonction d'onde $\Psi^{I}$ à l'instant $\theta+\theta^{\prime}$ si le photon n'avait jamais quitté l'atome $I$. Mais il est clair que maintenant $\theta+\theta^{\prime}$ peut s'étendre sur un intervalle de temps $2 \tau$. Tout se passe comme si l'on observait la résonance magnétique d'un atome $I$ dont la durée de vie serait double. La raie de résonance magnétique est dans ce cas 2 fois plus fine.

Ainsi, la diffusion cohérente peut conduire à des affinements sensibles quand on élève la tension de vapeur. En pratique on observe la superposition dans des proportions difficiles à évaluer, de diffusion simple, double cohérente, triple cohérente, etc... et de diffusion incohérente (entre isotopes différents par exemple). Le temps $T_{\mathrm{e}}$ est donc une quantité très différente du temps $\tau^{\prime}$ mesuré par les méthodes basées sur la survivance du rayonnement dans une cellule ; $\tau^{\prime}$ est nécessairement plus grand que $T_{\mathrm{e}}$ puisque la diffusion incohérente contribue pour $2 \tau$ dans $\tau^{\prime}$ et seulement pour $\tau$ (ou moins) dans $T_{\text {e }}$. Ce résultat est en complet accord avec les mesures d'Holstein [6] (si on les extrapole aux tensions de vapeur où nous opérons ) mais en contradiction avec celles de Garrett [5].

Il nous semble difficile, dans la méthode de Garrett, d'évaluer avec certitude à moins de $10 \%$ près la valeur absolue d'un temps de l'ordre de $10^{-7}$ sec, et l'on peut réconcilier ses résultats avec les nôtres si l'on ajuste à la même valeur les durées de vie que fournissent les 2 méthodes. En outre la géométrie des 2 montages n'était pas comparable.

La résonance optique est un processus cohérent. Il n'est pas surprenant que la diffusion multiple le soit aussi. Les 2 phénomè̀nes ont été analysés jl y a longtemps déjà par V. Weisskopf [15] qui a donné la justification théorique des propriétés de cohérence. Plus récemment, R. H. Dicke [16] a envisagé une situation analogue dans sa définition des états ( super-radiants ».

Il était connu qu'un atome éclairé par un mélange cohérent de photons $\sigma^{+}$et $\sigma$, réémet des photons $\sigma^{+}$et $\sigma$ en phase ; la propriété décrite ci-dessus n'est qu'une généralisation de ce phénomène.

Conclusion. - Nous pouvons dire en conclusion que (dans le cas de la vapeur de mercure), les phénomènes de dépolarisation et de survivance du rayonnement sont dus à la diffusion multiple de la lumière [17]. L'affinement de la raie de résonance magnétique quand la densité de vapeur s'élève, ne s'explique que dans l'hypothèse d'une diffusion multiple cohérente. Cette hypothèse doit permettre de calculer simultanément les courbes donnant le degré de polarisation et la largeur des raies de résonance magnétique en fonction du nombre d'atomes diffusants.

Appendice : Caleul de $\boldsymbol{T}_{\mathrm{e}_{0}}$. Détermination des paramètres. - Nous avons déterminé expérimentalement la manière dont varie pour l'isotope 202, dans le mercure naturel, l'intensité de la résonance magnétique (à résonance, $\Omega=0$ ) pour un niveau de radiofréquence $\gamma H_{1}$ donné, quand la température varie.

Les courbes donnant $I=b_{202} B_{202}\left(\gamma H_{1}, T_{\mathrm{e}_{202}}, 0\right)$ sont représentées sur la figure 9 , en fonction de log $N$ du nombre total d'atomes de mercure dans la cellule.

Chaque courbe est tracée pour un niveau de radio fréquence donné, les courbes les plus hautes correspondant aux intensités de radiofréquence les plus fortes. Ces courbes présentent un maximum qui se déplace vers les fortes valeurs de $N$ lorsque $\gamma H_{1}$ diminue. Autrement dit, pour un niveau de radiofréquence donné, il existe une température pour laquelle l'intensité de résonance magnétique est maximum. (La forme de ces courbes dépend évidemment de la géométrie de la cellule sur laquelle on opère).

La forme de ces courbes se comprend facilement. A basse température (en $\mathrm{B}$ ) la dépolarisation est faible mais il y a très peu d'atomes dans la cellule. A forte température, il y a beaucoup d'atomes mais 
la lumière est presque complètement dépolarisée. Il y a donc 2 effets variant en sens inverse, et par suite un optimum pour l'intensité de la résonance magnétique.

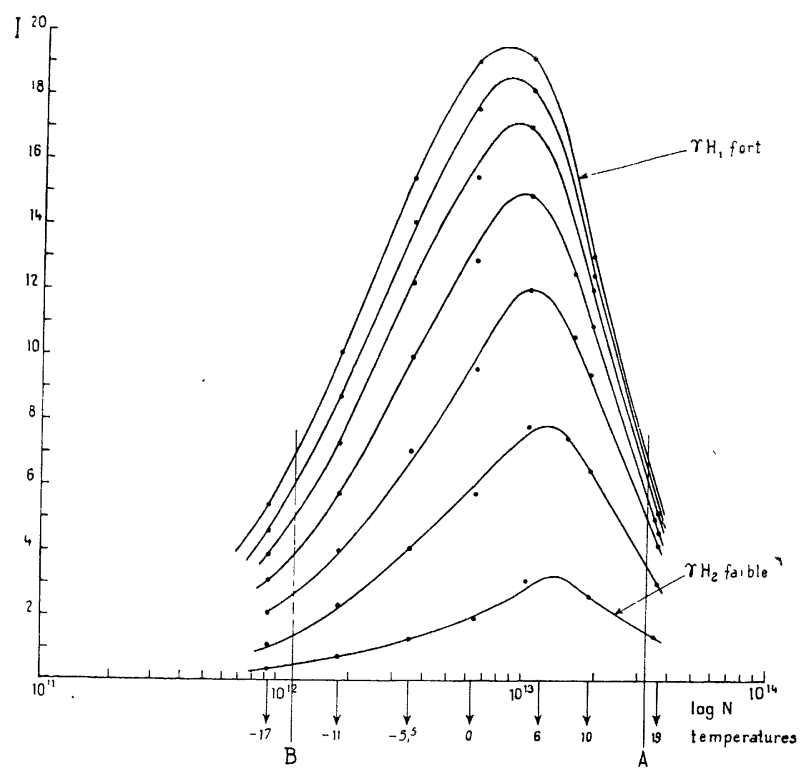

Fig. 9.

Il apparaît sur la figure 9 que les courbes sont beaucoup plus serrées en A (fortes valeurs de $N$ ) qu'en B. Ceci est un effet dû à la variation de $T_{\text {e }}$ avec la température. En B, $T_{\mathrm{e}}$ est court (résonances larges), et les intensités de radiofréquence utilisées sont insuffisantes pour saturer la raie dont l'intensité augmente de façon appréciable lorsqu'on augmente $\gamma H_{1}$. En A, au contraire, $T_{\mathrm{e}}$ est long (résonances fines) et les intensités de radiofréquence précédentes sont suffisantes pour saturer la raie dont l'amplitude ne change pratiquement plus quand $\gamma H_{1}$ couvre la gamme précédente (c'est ce même phénomène qui est à l'origine du déplacement du maximum vers les fortes valeurs de $N$ quand $\gamma H_{1}$ diminue). Ces courbes nous permettent de déterminer les valeurs relatives de $b_{\mathrm{j}}$ pour les divers isotopes. On opère de la manière suivante : A $0{ }^{\circ} \mathrm{C}$ par exemple, on mesure sur la courbe I,

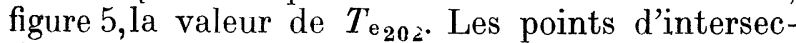
tion descourbes de la figure 9 avec la verticale correspondant au point 0 oC sont données par l'équation 2

$a_{202} b_{202} B_{202}\left(\gamma H_{1}, T_{\mathrm{e}_{202}}, 0\right) \equiv a_{202} b_{202} \frac{\left(\gamma H_{1} T_{\mathbf{e}_{202}}\right)^{2}}{1+4\left(\gamma H_{1} T_{\mathbf{e}_{202}}\right)^{2}}$.

$a_{202}$ et $b_{202}$ étant constants. On en déduit donc les valeurs de $\gamma H_{1}$ pour lesquelles ces courbes ont été tracées.

La courbe I, figure 5, permet de mesurer $T_{\text {ej }}$ pour les divers isotopes, par suite de calculer le paramètre $\gamma H_{1} T_{\mathrm{e}_{\mathrm{j}}}$ et en fin

$$
B_{\mathrm{j}}\left(\gamma H_{1}, T_{\mathrm{ej}}, 0\right) \equiv \frac{\left(\gamma H_{1} T_{\mathrm{ej}}\right)^{2}}{1+4\left(\gamma H_{1} T_{\mathrm{ej}}\right)^{2}} .
$$

Revenant aux courbes de la figure 9 , on mesure (pour la valeur convenable de $\gamma H_{1}$ ) la quantité $b_{\mathrm{j}} B_{\mathrm{j}}\left(\gamma H_{1}, T_{\mathrm{e}_{\mathrm{j}}}, 0\right)$ en se plaçant à la concentration appropriée de l'isotope envisagé. On en déduit $b_{\mathrm{j}}$. (La valeur obtenue ne doit pas dépendre de la valeur choisie pour $\gamma H_{1}$, ce qui se vérifie très bien. On peut mesurer d'ailleurs directement sur les courbes 9 le paramètre $\gamma H_{1} . T_{\mathrm{ej}}$. Les valeurs concordent bien avec les précédentes.)

Le procédé précédent est valable pour l'isotope 200 mais ne l'est plus pour les isotopes 198 et 204. Les raies de résonance de cas derniers coïncident avec des composantes hyperfines des isotopes impairs. Par suite, les effets d'absorption, inclus dans le paramètre $b_{\mathrm{j}}$ sont plus forts que ce qu'indique la méthode précédente. Tout se passe comme si la concentration de l'isotope 198 était plus forte que sa vraie valeur en ce qui concerne la détermination du paramètre $b_{198}$ mais avait sa vraie valeur en ce qui concerne la détermination du paramètre $T_{\mathrm{e}_{\mathbf{1 9 8}}}$.

Nous avons alors tracé directement la courbe donnant

$$
I_{198}=a_{198} b_{198} B\left(\gamma H_{1}, T_{\mathrm{e}_{198}}, 0\right)
$$

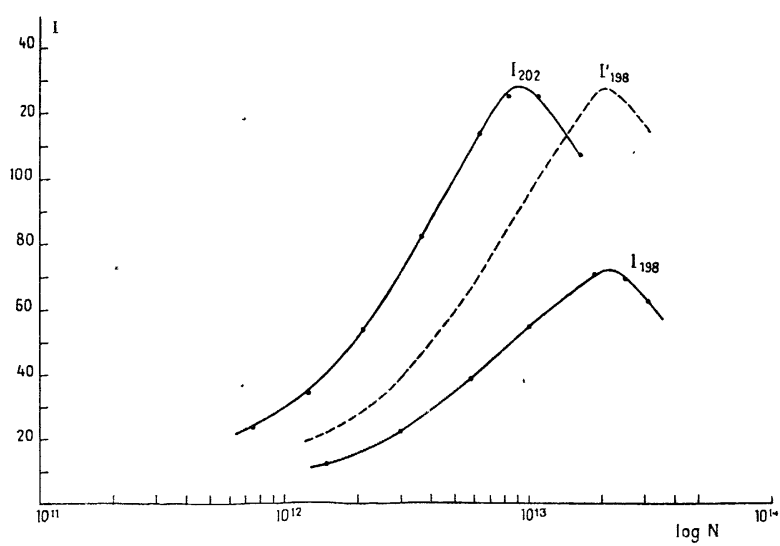

FIG. 10.

en fonction de $\log N$. La figure 10 donne le résultat. Nous avons tracé $I_{198}$ et $I_{202}$ (sources excitatrices ${ }^{198} \mathrm{Hg}$ puis ${ }^{202} \mathrm{Hg}$ - lampe de résonance contenant du mercure naturel) en utilisant la même intensité de radiofréquence. (Le rapport d'intensité des sources excitatrices n'est pas connu.)

On voit que l'optimum est obtenu pour ${ }^{198} \mathrm{Hg}$ à une température beaucoup plus élevée que pour ${ }^{202} \mathrm{Hg}$.

On peut normaliser la courbe $I_{198}$ de manière qu'elle ait même intensité au maximum que $I_{202}$ (courbe en pointillé marquée $I_{198}^{\prime}$ ). On constate que la courbe $I^{\prime}{ }_{198}$ ne se déduit pas de $I_{202}$ par une translation. Si l'on fait une translation qui fait coïncider les maxima, le reste des courbes diffère, mais les différences bien que réelles, demeurent 
toujours farbles. La valeur de cette translation ne correspond pas, bien sûr, à la concentration isotopique de ${ }^{198} \mathrm{Hg}$, mais à une concentration qui est naturellement plus forte que la valeur vraie. C'est cette concentration fictive que nous avons adoptée pour déterminer $b_{198}$ par la méthode décrite précédemment.

Il serait souhaitable de connaître le rapport d'intensité des sources 198 et 202 pour lesquelles nous avons opéré. Mais la mesure n'est pas simple puisque la forme des raies émises compte au premier chef.

${ }^{204} \mathrm{Hg}$ étant très peu concentré $(6 \%)$ et jouant un rôle assez faible, nous avons traité son cas de manière identique à celle utilisée pour l'isotope 200 . Cela est gravement erroné mais n'affecte pas beaucoup le résultat final.

Cet article sera suivi d'une étude théorique publiée par M. J. P. Barrat.

Manuscrit reçu le 3 décembre 1956.

\section{IRÉFÉRENGES}

[1] Voir par exemple : Mitchell (A. G. G.) et ZemansKy (M. W.), Resonance radiation and excited atoms, Cambridge University Press, 1934. Pringsheim (P.), Fluorescence and phosphorescence, Interscience Publishers Inc., New-York, 1949.

[2] Wood (R. W.), Phil. Mag., 1922, 44, 1109. Rayleigh, Proc. Roy. Soc., 1922, 102, 190.

[3] Keussler (V. von), Ann. Physik, 1927, 82, 793.

[4], Voir référence [1]: Mitchell et Zemansky, page 312. Pringsheim, page 102.

[5] Garrett (P. H.), Phys. Rev., 1932, 40, 779.

[6] Milne (E. A.), J. London Math. Soc., 1926, 1, 1. Holstein (T.), Phys. Reo., 1947, 72, 1212. HolStein (T.), Alpert (D.) et McGoubrey (A. O.), Phys. Rev., 1949, 76, 1257 ; Phys. Rev., 1951, 82, 567 ; Phys. Rev., 1952, 85, 985.

[7] Buhr, Z. Physik, 1938, 109, 180.

[8] Voir Mitchell (A. G. G.) et Zemansky (M. W.), page 147 où se trouvent de nombreuses références.
[9] Cojan et Lennuier (R.), C. R. Acad. $S c ., 1950,231$, 1450.

[10] Brossel (J.) et Bitter (F.), Phys. Rev., 1952, 86, 308. Brossel (J.), Ann. Physique, 1952, 7, 622.

[11] Majorana (E.), Nuovo Cimento, 1932, 9, 43.

[12] Ladenburg (R.) et Wolfson (G.), Z. Physik, 1930, $63,616$.

[13] Lorentz (H. A.), Proc. Amst. Acad., 1915, 18, 134.

[14]. Hanle (W.), Z. Physik, 1924, 30, 93. Hanle (W.), Ergeb der. Exakten Naturwiss., 1925, 4, 214. WEISskopf (V.), Ann. Physik, 1931, 9, 23. Breit (G.), Rev. Mod. Physics, 1933, 5, 91.

[15] Weiss kop F (V.), Ann. Physik, 1931, 9, 23.

[16] Dicke (R. H.), Phys. Rev., 1954, 93, 99.

[17] Rollet (N.), Brossel (J.), Kastler (A.), $C . R$. Acad. Sc., 1956, 242, 240. 